\title{
Temporal variability in the Doppler-shift of solar transition region lines
}

\author{
J. G. Doyle ${ }^{1}$, M. S. Madjarska ${ }^{1}$, I. Roussev ${ }^{1,2}$, L. Teriaca $^{3}$, and J. Giannikakis ${ }^{4}$ \\ 1 Armagh Observatory, College Hill, Armagh, BT61 9DG, N. Ireland \\ 2 Center for Space Environment Modeling, 1414 Space Research Building, The University of Michigan, \\ Ann Arbor, MI 48109-2143, USA \\ 3 Osservatorio Astrofisico di Arcetri, Largo Enrico Fermi 5, 50125 Firenze, Italy \\ ${ }^{4}$ Sect. of Astrophysics, Astronomy and Mechanics, Dept. of Physics, Univ. of Athens, Athens 15783, Greece \\ e-mail: madj@star.arm.ac.uk; iroussev@umich.edu; 1te@arcetri.astro.it
}

Received 19 December 2001 / Accepted 19 September 2002

\begin{abstract}
High cadence datasets taken in C III $977 \AA$ A, O vi $1032 \AA$ and Ne vIII $720 \AA$ were analysed in an effort to establish the extent of the variability in the Doppler-shift of typical mid-transition region lines. The shortest time-scale variability seems to occur in the network boundary regions where the line-shift can vary by $7-8 \mathrm{~km} \mathrm{~s}^{-1}$ in less than $1 \mathrm{~min}$. The internetwork region also shows variability although this tends to be longer lived, $\sim 2-3 \mathrm{~min}$. The average line-shift in $\mathrm{C}$ III is a red-shift which ranges from $\sim 2 \mathrm{~km} \mathrm{~s}^{-1}$ to $\sim 20 \mathrm{~km} \mathrm{~s}^{-1}$ with an average value for all regions selected being around $10 \mathrm{~km} \mathrm{~s}^{-1}$ in very good agreement with that derived by others. The red-shift values indicate a clear difference between network and internetwork regions, with the largest red-shift being present at the network boundary. For $\mathrm{O} v \mathrm{vI}$, this gives an average red-shift ranging from 5 to $10 \mathrm{~km} \mathrm{~s}^{-1}$. For Ne vIII, there is a $13 \mathrm{~km} \mathrm{~s}^{-1}$ difference between internetwork and bright network plasma with the bright network being more red-shifted. This could imply that the bright network regions are dominated by spicule down-flow.

In the second part we present results from 2-dimensional (2D) dissipative magnetohydrodynamic (MHD) simulations of the response of the solar transition region to micro-scale energy depositions. A variety of temperatures at which the energy deposition takes place as well as the amount of energy deposited are examined. This work is a continuation of previous related simulations where small-scale energy depositions were modelled in 1D hydrodynamics. The observable consequences of such transient events are then computed for three transition region lines, namely C IV $1548 \AA$, O VI $1032 \AA$, and Ne VIII $770 \AA$, under the consideration of non-equilibrium ionization.
\end{abstract}

Key words. Sun: atmosphere - Sun: transition region - Sun: activity

\section{Introduction}

It is now well established that spectral lines formed at around $100000 \mathrm{~K}$ in the solar transition region exhibit a net red-shift. If this observed red-shift can be attributed to plasma down-flow, it should be very important from the point of the transition region dynamics and may have implications for the energy balance in the outer solar atmosphere. Models attempting to explain the net mass flows in magnetic loops include those where the flows are driven by asymmetries (such as heating or pressure imbalances) between the two legs of the loop (Mariska \& Boris 1983; Mariska 1988; Spadaro et al. 1991) or by radiatively-cooling condensations (Reale et al. 1997). Another model suggests that the observed red-shift could be due to the return of spicular material (Pneuman \& Kopp 1978). This model has recently been re-evaluated by Wilhelm (2000).

Send offprint requests to: J. G. Doyle, e-mail: jgd@star.arm.ac.uk
Hansteen (1993) showed that red-shifted transition region lines are predicted for downward propagating acoustic waves, and blue-shifted lines are predicted when the perturbations propagate towards the corona (Hansteen \& Wikst $\varnothing 1$ 1994). A followup study by Hansteen et al. (1977) give additional simulation results using nonlinear Alfven waves which give larger line-shifts. In general, impulsive energy release (nano/microflaring), at the top of magnetic loops can generate downward traveling MHD waves, perhaps steepening into shocks and leading to both red- and blue-shifts, depending on the line formation temperature. Recently, Chae et al. (1997) showed that the enthalpy flux due to down-flow determines the overall energy balance in the transition region at temperatures below $10^{5} \mathrm{~K}$.

In a recent observational paper, we looked at the behaviour of spectral lines from the chromosphere to the corona (Teriaca et al. 1999a). It was found that the Doppler velocities ranged from $\sim 0 \mathrm{~km} \mathrm{~s}^{-1}$ at $\sim 20000 \mathrm{~K}$ to a red-shift of 

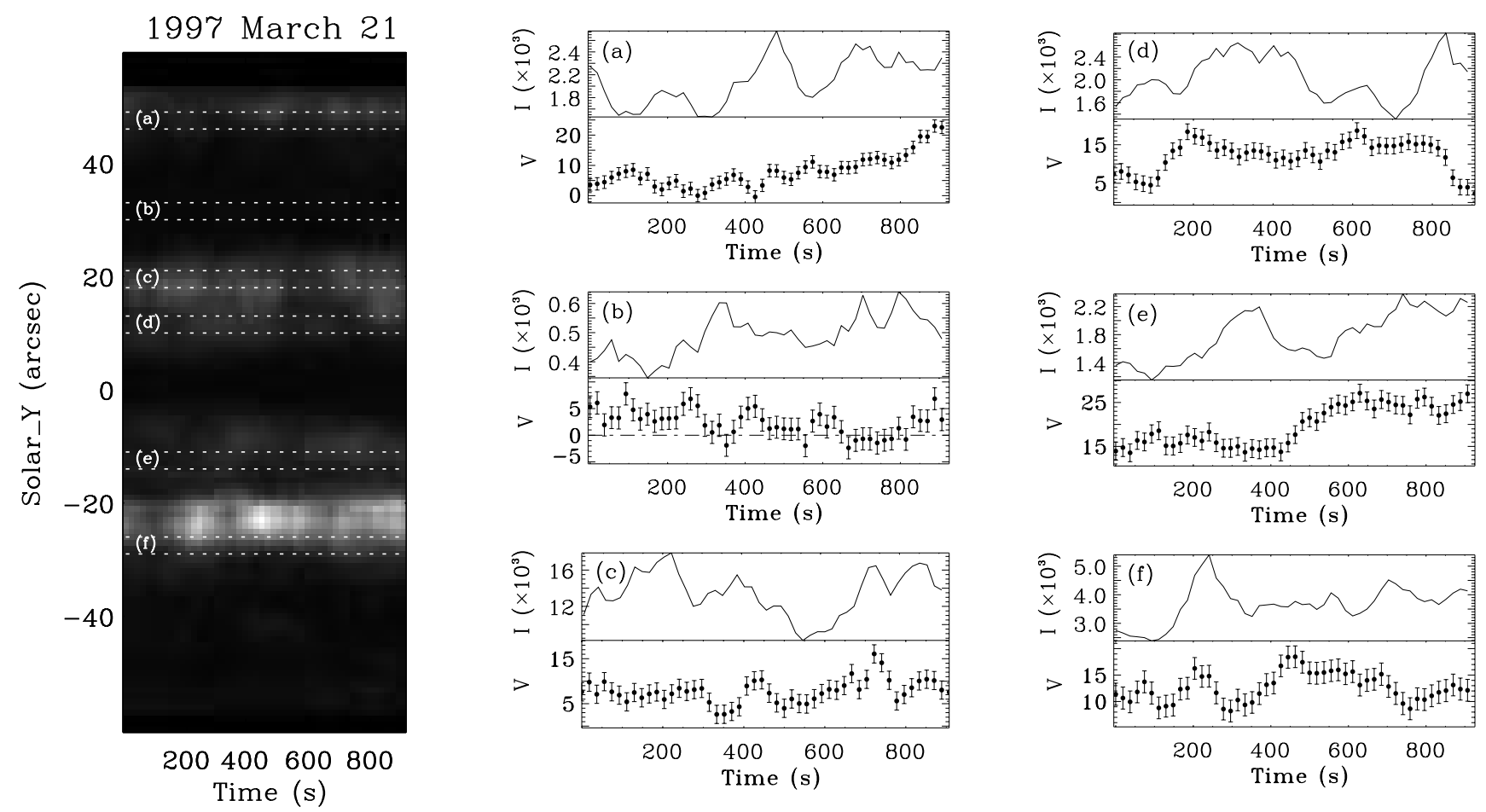

Fig. 1. Line radiance variations (erg cm $\mathrm{cm}^{-2} \mathrm{~s}^{-1} \mathrm{sr}^{-1}$ ) and Doppler shift $\left(\mathrm{km} \mathrm{s}^{-1}\right)$ of the $\mathrm{C}_{\mathrm{III}} 977 \AA$ A . An integration along the slit (left panel) was performed over 4 pixels (as labeled). The left panel image represents the intensity along the slit. The data have a cadence of $\sim 18 \mathrm{~s}$.

$10 \mathrm{~km} \mathrm{~s}^{-1}$ at $1.9 \times 10^{5} \mathrm{~K}$ for the "quiet Sun", and to $\sim 15 \mathrm{~km} \mathrm{~s}^{-1}$ at $10^{5} \mathrm{~K}$ for an active region. At higher temperature an opposite behaviour was observed. In the "quiet" Sun a blue-shift of $\sim-2 \mathrm{~km} \mathrm{~s}^{-1}$ was derived at the Ne vIII formation temperature $\left(6 \times 10^{5} \mathrm{~K}\right)$, while in the active region, a blue-shifted value around $-8 \mathrm{~km} \mathrm{~s}^{-1}$ was observed for the same spectral line.

The above observational work was, however, time-averaged and did not cover the important area of time variability. Doyle et al. (1998) found periods approaching $\sim 5 \mathrm{~min}$ to be present in lines due to $\mathrm{O}$ III, $\mathrm{O}$ IV and $\mathrm{O}$ v. In one dataset a period approaching $10 \mathrm{~min}$ was present for $\sim 40 \mathrm{~min}$. The size of these emitting features was limited to a few arcsec. In all datasets examined, there was excess power below $4 \mathrm{mHz}$ everywhere along the slit, although the observed periods did not always come from the most intense regions. Banerjee et al. (2001) in an analysis of network data detected intensity as well as velocity oscillations for very small regions of the network boundary, for a wide range of temperature lines starting from the low chromosphere, $\mathrm{N}$ I, to the transition region, $\mathrm{O}$ VI. The periods were often intermittent, occurring only during part of the full time sequence. Wikstøl et al. (2000) showed that in the internetwork there was a phase shift relation between the intensity of the continuum around $1043 \AA$ and the $\mathrm{O}$ vi line shifts that could indicate the presence of upward propagating waves. In the network, the existence of an opposite phase relation between $\mathrm{C}$ II and $\mathrm{O}$ vi line shifts, indicated that waves may propagate downwards in these regions. It was also noted that $\mathrm{C}_{\text {II }}$ and $\mathrm{O}$ vi line profiles reveal, on statistical average, more red-shifted emission in the network than in the internetwork lanes. A similar result was also found by Hansteen et al. (2000). Recently, a detailed analysis of transition region line profiles performed by Peter $(2000,2001)$ brought further support to the hypothesis that different mechanisms may dominate the transition region dynamics in the network and internetwork regions. Thus, any attempt to study the temporal and spatial behaviour of spectral lines originating from transition region temperatures should also distinguish between the network and internetwork regions.

Teriaca \& Doyle (2001) presented a comparison of observations versus numerical simulations of the response of the solar atmosphere to an energy perturbation of $4 \times 10^{24}$ ergs representing an energy release during magnetic reconnection in a 1D semi-circular flux tube. The temporal evolution of the thermodynamic state of the loop were converted into C III 977, C Iv 1548, O v 630, O vi 1032, Ne vII 465 and Ne vIII 770 line profiles via non-equilibrium calculation of ion populations. Performing an integration over the entire period of simulation, red-shifts of 8.5, 6.1 and $1.7 \mathrm{~km} \mathrm{~s}^{-1}$, were found for $\mathrm{C}$ III, $\mathrm{C}$ IV, and $\mathrm{O} v$ while blue-shifts of $-1.8,-3.9$ and $-10.7 \mathrm{~km} \mathrm{~s}^{-1}$ were derived for $\mathrm{O}$ VI, $\mathrm{Ne}$ VII and $\mathrm{Ne}$ VIII respectively, in good agreement with observations.

The objective of the present paper is two-fold, in the first part we present further evidence via selected high cadence observational sequences that the Doppler-shift is highly time dependent, down to a few seconds for lines formed over a large fraction of the transition region. For this we choose "quiet" Sun datasets taken in C III $\sim 977 \AA$, O vI $\sim 1032 \AA$ and Ne VIII $\sim 770 \AA$ obtained by the SUMER instrument on-board the SoHO satellite. The region observed included cells, network areas, and the boundaries between them. 

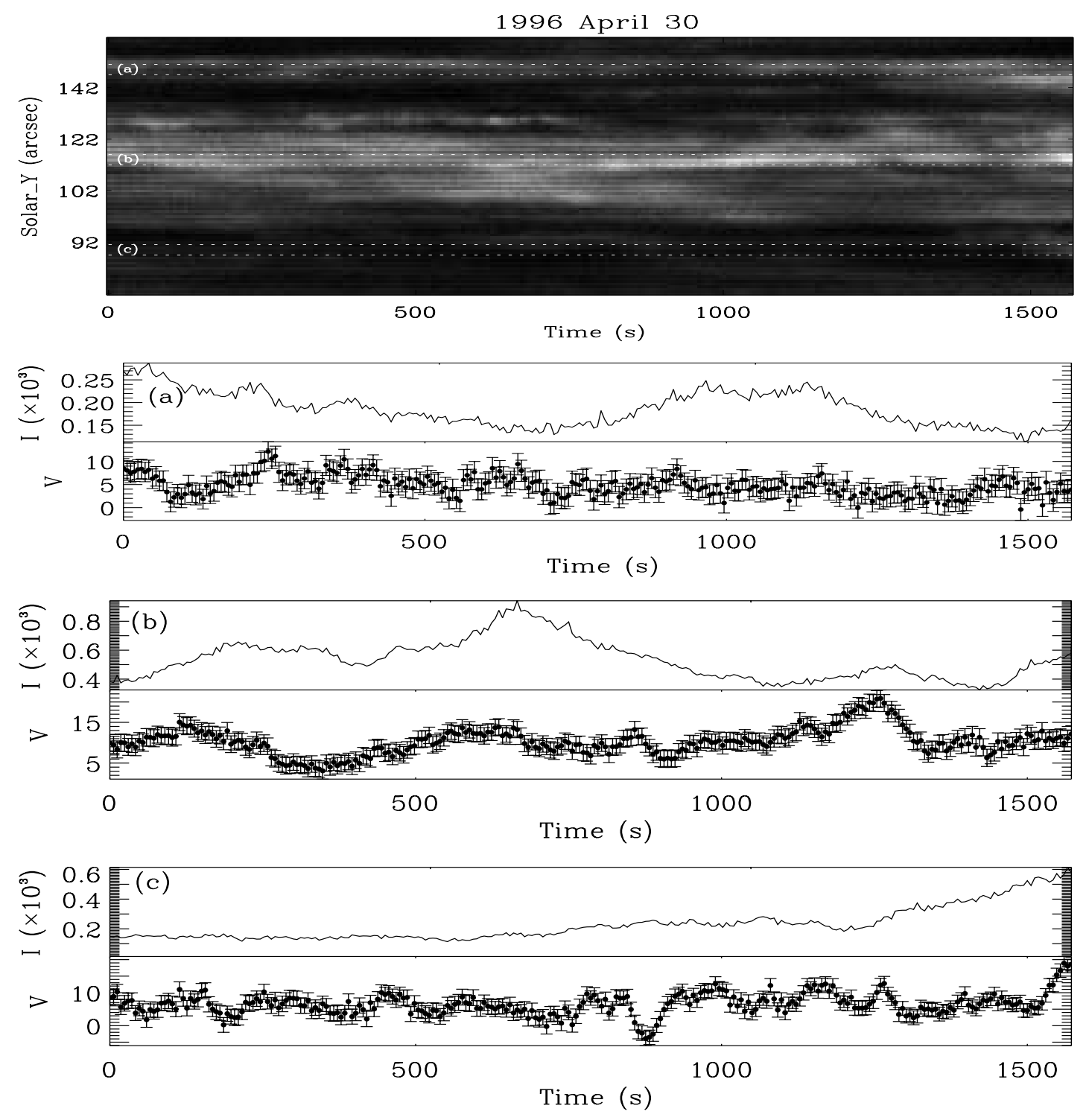

Fig. 2. Line radiance variations ( $\mathrm{erg} \mathrm{cm}^{-2} \mathrm{~s}^{-1} \mathrm{sr}^{-1}$ ) and Doppler shift $\left(\mathrm{km} \mathrm{s}^{-1}\right)$ of the $\mathrm{O}$ vi $1032 \AA$. In all cases, an integration along the slit (see top panel) was performed over 5 pixels.

In the second part we examine the observable consequences, in terms of line profiles, of small-scale energy depositions of different magnitudes taking place at different heights in a 2D "quiet" Sun transition region environment and whether such a model can explain both the variability and the direction of the observed line-shift. To this end, the equilibrium initial state is perturbed by an ad hoc heating term in the energy equation. The underlying motivation to choose small-scale energy depositions instead of driving magnetic reconnection (like in the reconnection studies of Roussev et al. 2001a,b) was to use the energy that goes to heat the plasma as an input parameter, instead of an output parameter (i.e. a reconnection model). The idea is to study the value of Doppler shift as a function of the energy input. For this, we choose three different values for the total amount of energy release, $E_{0}$, i.e., $4 \times 10^{24} \mathrm{ergs}$, $1 \times 10^{25} \mathrm{ergs}$, and $4 \times 10^{25} \mathrm{ergs}$, respectively. These values could, in principle, represent a nano-flare event as a result of an ongoing magnetic reconnection. Each of these energy depositions is then considered to occur at four different plasma temperatures, i.e., $5 \times 10^{4} \mathrm{~K}, 1 \times 10^{5} \mathrm{~K}, 2 \times 10^{5} \mathrm{~K}$, and $4 \times 10^{5} \mathrm{~K}$, respectively.

Roussev et al. (2001a,b) recently presented a numerical study relevant to explosive events, where 2D MHD calculations were converted into line profiles representative of transition region lines. The calculations of ion populations allow for departures from equilibrium ionization, which proved to be important since these dynamic events involve time scales smaller than the ionization and recombination times. We adopt this procedure in these simulations.

\section{Observations and data reduction}

SUMER is a normal incidence spectrograph operating over the wavelength range $\sim 500$ to $1610 \AA$ (details can be obtained from Wilhelm et al. 1995; Lemaire et al. 1997). The datasets selected 

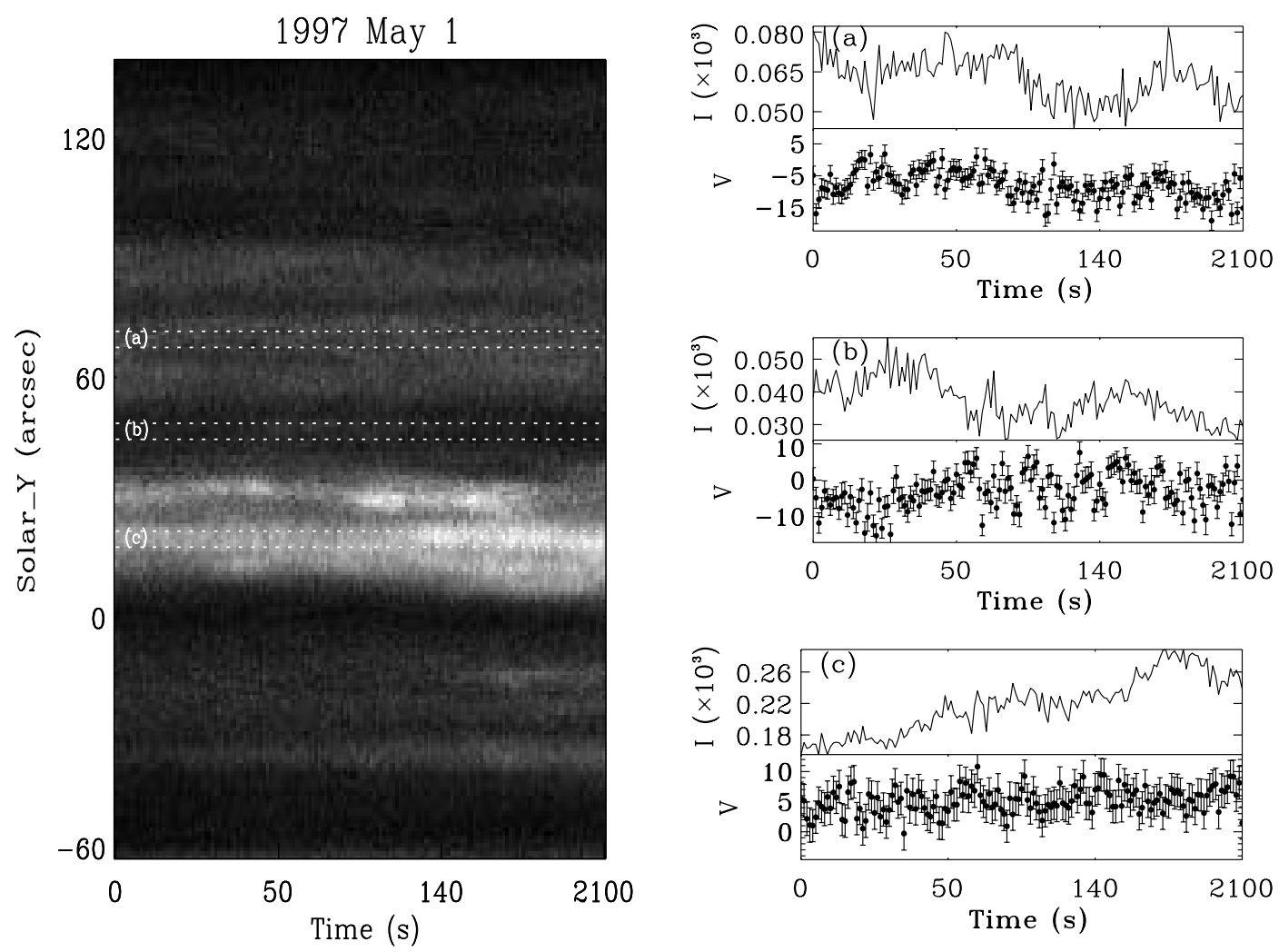

Fig. 3. Line radiance variations (erg cm ${ }^{-2} \mathrm{~s}^{-1} \mathrm{sr}^{-1}$ ) and Doppler shift $\left(\mathrm{km} \mathrm{s}^{-1}\right)$ of the Ne vIII $770 \AA$ A. An integration along the slit (left panel) was performed over 4 pixels (as labeled). The left panel image represents the intensity along the slit. The data have a cadence of $\sim 14 \mathrm{~s}$.

were obtained on March 21, 1997 (C III $977 \AA$ A), April 30, 1996 (O vi $1032 \AA$ ) and May 1, 1997 (Ne vIII $770 \AA$ ) on the "quiet" Sun in a sit-and-stare mode with no compensation of solar rotation applied (although many other high cadence datasets exist, only a few of these have a corresponding full spectrum which is required for absolute wavelength calibration). For the $\mathrm{C}$ III line, slit $8\left(0.3 \times 120 \operatorname{arcsec}^{2}\right)$ was used with detector B with an integration time of $2.5 \mathrm{~s}$ from 18:23:40 to 18:39:09 UT. However, the electronics readout time leads to an effective observing cadence of $\sim 6 \mathrm{~s}$. The short integration time combined with the narrow slit ensured that the count-rate for this strong line remained below the non-linear limit of $10 \mathrm{cts} \mathrm{s}^{-1}$ pixel $^{-1}$. Since the slit is only 0.3 arcsec wide, in $15 \mathrm{~min}$ it covers, due to the absence of solar rotation compensation, approximately a 3 arcsec region on the Sun. The $\mathrm{O}$ vi line was registered on detector A using slit $4\left(1 \times 120 \operatorname{arcsec}^{2}\right)$ with an exposure time of 6 s from 22:29:01 to 22:55:20 UT. The Ne viII line was taken using slit $2\left(1 \times 300 \operatorname{arcsec}^{2}\right)$ on detector B exposing for $7 \mathrm{~s}$ from $20: 49$ to $22: 48 \mathrm{UT}$.

The reduction of SUMER raw images followed several stages, i.e., local gain correction, flat-field subtraction, a correction for geometrical distortion and radiometric calibration (providing the conversion from counts $\mathrm{px}^{-1} \mathrm{~s}^{-1}$ to Watt $\left.\mathrm{m}^{-2} \mathrm{sr}^{-1} \AA^{-1}\right)$. The signal to noise level is determined by the photon statistics.

The position of the SUMER slit during the C III and Ne vIII observations was set on heliographic coordinates $X=0^{\prime \prime}$, $Y=0^{\prime \prime}$. As can be seen from Figs. 1-3 (see text below), the slit covered several network and internetwork structures in each dataset. In order to increase the S:N (signal-to-noise) level, in the $\mathrm{C}$ III case we binned over three consecutive spectra (leading to a time resolution of $\sim 18 \mathrm{~s}$ ) and over 4 spatial pixels (giving a spatial resolution of 4 arcsec along the slit). Different locations along the slit were analysed, representative of a variety of different features. The $\mathrm{O}$ vi dataset consists of 1024 images centered on heliographic coordinates $X=199^{\prime \prime}$, $Y=102^{\prime \prime}$. An integration along the slit over five pixels was performed in order to improve the signal-to-noise ratio. The evolution of 20 regions along the slit was studied. For the Ne vIII dataset, we binned over two spectra (obtaining a temporal resolution of $14 \mathrm{~s}$ ) and 4 spatial pixels. For all datasets, there was no rotational compensation applied and therefore it is important to note that after $390 \mathrm{~s}$ the slit is no longer pointing at the same region.

Since we are interested in absolute line shifts, an absolute wavelength calibration is vital. On SUMER there is no onboard calibration source, so the wavelength calibration is usually done using chromospheric lines of neutral atoms. A full spectrum image with an exposure time of $300 \mathrm{~s}$ was taken just before the temporal series in $\mathrm{C}$ III allowing us to perform the wavelength calibration. For the $\mathrm{O}$ vi dataset, one half detector image with an exposure time of $150 \mathrm{~s}$ is available. The dispersion relation was calculated using $\mathrm{O}$ I reference lines (for details see Teriaca et al. 1999a). The situation for the Ne VIII line was not optimum since the data were registered in first order on detector B and therefore with the lack of chromospheric 
lines, an "absolute" calibration is not possible. Moreover, since the data were taken at disk center, the other used method of an off-limb average zero Doppler shift can not be applied (see Sect. 3.3).

\section{Discussion on the observational results}

\section{1. $C$ III $977 \AA$}

The regions sampled (see Fig. 1) include: (a) the network region, (b) an internetwork region, (c) a network region, (d) a network boundary, (e) a network region and (f) the boundary of a bright network. All of the sampled 6 regions showed short time-scale variability in the absolute line-shift.

The shortest time-scale variability seems to occur in the network boundary regions where the line-shift can vary by $7-$ $8 \mathrm{~km} \mathrm{~s}^{-1}$ in less than $1 \mathrm{~min}$. The internetwork region also shows variability although this tends to be smaller. The average lineshift in all regions is for a red-shift, ranging from $8 \mathrm{~km} \mathrm{~s}^{-1}$ in (a), $2 \mathrm{~km} \mathrm{~s}^{-1}$ in (b), $7 \mathrm{~km} \mathrm{~s}^{-1}$ in (c), $12 \mathrm{~km} \mathrm{~s}^{-1}$ in (d), $20 \mathrm{~km} \mathrm{~s}^{-1}$ in (e) and $13 \mathrm{~km} \mathrm{~s}^{-1}$ in (f). These figures indicate a clear difference between network and internetwork regions, with the largest red-shift being present at the network boundary. Only in the internetwork, did the $\mathrm{C}$ III line show some evidence for a blue-shift. Hansteen et al. (2000) reported that the internetwork showed on average a $4 \mathrm{~km} \mathrm{~s}^{-1}$ red-shift.

There is no evidence for high frequency oscillations in either the intensity or the velocity in the $\mathrm{C}$ III data and since the dataset is only $\sim 15 \mathrm{~min}$ long, oscillations as detected by Doyle et al. (1998) were not observable. The data, however, is highly variable, which could be perhaps interpreted as a series of small energy releases, as distinct from explosive events which are ruled out due to the lack of non-Gaussian line profiles.

\subsection{O VI $1032 \AA$}

The regions sampled in the $\mathrm{O}$ vi datasets include (a) a weak network region, (b) a strong network region and (c) an internetwork region (see Fig. 2). The average velocities in each of these indicate a net red-shift of (a) $4.8 \mathrm{~km} \mathrm{~s}^{-1}$, (b) $10 \mathrm{~km} \mathrm{~s}^{-1}$ and (c) $6.5 \mathrm{~km} \mathrm{~s}^{-1}$. As in the $\mathrm{C}$ III dataset, there are locations which show quite fast changes, e.g. in region (c) we see a sharp decrease in the line-shift from $10 \mathrm{~km} \mathrm{~s}^{-1}$ to $-5 \mathrm{~km} \mathrm{~s}^{-1}$ and finally back to $10 \mathrm{~km} \mathrm{~s}^{-1}$ in less than $60 \mathrm{~s}$. The strong network locations show a gradual change in the line-shift occurring over an interval of $\sim 200-300 \mathrm{~s}$. Note that the time of peak red-shift does not necessary correspond to the time of peak intensity. Furthermore, at various times/locations in the strong network region, the line-shift approaches zero although, as seen from the values quoted above, the largest time-averaged net red-shift corresponds to the strong network feature. Note that the spatial region covered by the slit in the $\mathrm{E}-\mathrm{W}$ direction is $\sim 4$ arcsec compared to the slit width of 1 arcsec.

\section{3. $\mathrm{Ne}$ VIII $770 \AA$}

The regions sampled (see Fig. 3) include: (a) a network region, (b) an internetwork region and (c) a bright network boundary.
In order to interpret our results we had to rely on measurements of Doppler shifts given in the literature. Brekke et al. (1997) and Chae et al. (1998) found a red-shift of $5 \pm 1.5 \mathrm{~km} \mathrm{~s}^{-1}$. These studies, however, adopted the vacuum wavelength of of $770.409 \AA$ for Ne viII. Dammasch et al. (1999) deduced the solar wavelength for this line for different regions on the Sun and derived a rest wavelength of $770.428 \pm 0.003$. Their rest wavelength determination was based on the assumption of no average Doppler shift above the solar limb and a comparison with laboratory wavelengths of cool chromospheric lines. They obtained an outflow from a coronal hole with a velocity of $9 \mathrm{~km} \mathrm{~s}^{-1}$, while for the "quiet" Sun the average velocity gave a blue-shift of $1 \pm 1 \mathrm{~km} \mathrm{~s}^{-1}$. Teriaca et al. (1999a) and Peter \& Judge (1999) found similar results of $-1.9 \pm 2.0 \mathrm{~km} \mathrm{~s}^{-1}$, using the chromospheric lines method and $-2.5 \pm 1.1 \mathrm{~km} \mathrm{~s}^{-1}$ applying the off-limb zero Doppler shift assumption, respectively.

The Doppler shifts of Ne vIII given in this work represent a relative value applying a spectral pixel size of $44.73 \mathrm{~m} \AA$ (W. Curdt, private communication). The relative wavelength is obtained from a single Gaussian fit of an average line profile over the whole time series. Despite the fact that the routine for geometric corrections was applied, we sampled regions which are close enough along the slit to avoid introducing errors due to geometric distortions.

As can be clearly seen from Fig. 3, there is a large difference between the velocities in the three regions. In the network we have an average velocity of $-8 \mathrm{~km} \mathrm{~s}^{-1}$, while the internetwork gives $-3 \mathrm{~km} \mathrm{~s}^{-1}$ and the bright network $+5 \mathrm{~km} \mathrm{~s}^{-1}$. Within each of these regions, the variation can be as large as $\pm 8 \mathrm{~km} \mathrm{~s}^{-1}$.

As in the $\mathrm{C}$ III and $\mathrm{O}$ vI datasets, such short time-scale variability could be interpreted as small energy releases. In the following sections, we enquire whether such small-scale energy depositions can produce observable Doppler shift in our lines.

\section{Physical model}

\subsection{Initial configuration}

We consider the same 2D physical model approximating the solar transition region as that presented in Roussev et al. (2001a). Refer to this earlier study for a detailed description of the governing MHD equations, normalization units, etc. We allow for a shallow temperature gradient along the field lines, and thus the initial configuration is more like a "cool loop" (see Mariska 1992). For the sake of simplicity, the effects of gravity are neglected, and therefore the 2D atmosphere presented below is not stratified. In terms of normalized physical variables, the initial state is:

$\boldsymbol{u}=0$

$b_{x}=0, b_{y}=\tanh \left(\delta x^{*}\right)$,

$e^{*}=\frac{1}{2(\gamma-1)}\left(1+\beta-b_{y}^{2}\right)$,

$\rho^{*}=\left[\frac{\left(1+\beta-b_{y}^{2}\right)}{\beta}\right]^{\theta}\left\{1+\Delta \rho\left\{1-\tanh \left[\epsilon\left(y^{*}+y_{\mathrm{s}}^{*}\right)\right]\right\}\right\}$, 
and

$T^{*}=(\gamma-1) \frac{e^{*}}{\rho^{*}}$

Here $e$ is the thermal energy, $\rho$ the mass density, $\gamma$ the adiabatic index, $\beta$ is the ratio of the gas pressure to the magnetic pressure, $\delta$ is a model parameter used to control the width of the current concentration, $\epsilon$ is the steepness of the transition region and $\theta$ is a free parameter (one over the polytropic index) which defines the density profile across the current concentration (i.e., in the $x$-direction). Also, the parameter $\Delta \rho$ defines the density (and also temperature) jump across the transition region, $2 \Delta \rho+1$. Values of all these parameters used in the present modelling are given in Roussev et al. (2001a).

We further assume an energy balance for the initial state, which is used to derive the 2D spatial distribution of the volumetric heating sources,

$S^{*}=\nabla^{*} \cdot \boldsymbol{q}^{*}+L_{\mathrm{r}}^{*} ;$ at $t^{*}=0$

where $S^{*}, q^{*}$ and $L_{\mathrm{r}}$ are the volumetric heating rate, fieldaligned conductive flux, and radiative losses, respectively. This way, apart from referring to a static equilibrium state in the initial background plasma, we also ensure there are no heating or cooling sources throughout the computational domain at time zero.

In Fig. 4 we show the physical variables for the initial state, i.e. the mass density, temperature, thermal energy, current density and magnetic field topology.

\subsection{Model of small-scale energy depositions}

In the present study, the localized energy release is placed at the current concentration and is assigned for a finite time. The explicit form of this additional heating term is chosen as

$E_{\mathrm{dep}}=E_{0} \alpha F\left(t^{*}\right) \exp \left\{-\left[\frac{x^{* 2}+\left(y^{*}-y_{0}^{*}\right)^{2}}{\sigma}\right]\right\}$,

where

$F\left(t^{*}\right)= \begin{cases}t^{*} / t_{\text {rise }}^{*} ; & 0<t^{*} \leq t_{\text {rise }}^{*}, \\ \exp \left[-\alpha\left(t^{*}-t_{\text {rise }}^{*}\right)\right] ; & t_{\text {rise }}^{*}<t^{*}\end{cases}$

Here $E_{0}$ is the total amount of energy release and $\alpha=\ln (10) / t_{90}^{*}$ (where $t_{90}^{*}$ represents the time during which $90 \%$ of the total energy deposition is released). Equation (8) describes a linear increase in the additional heating rate up to $t^{*}=t_{\text {rise }}^{*}$, followed by an exponential decay in time. The distribution of the energy release in space is chosen as a symmetric double Gaussian centered at $\left(0, y_{0}^{*}\right)$. The free parameter $y_{0}^{*}$ is used to move the position of energy deposition along the current concentration so that this takes place at different initial plasma temperatures. The parameters controlling the shape of the temporal and spatial distribution of the energy release are $\alpha$ and $\sigma$. As to the latter, $\sigma=M^{2} / \ln 2$, where $M$ is the full width at half maximum (see Sarro et al. 1999). The parameter $\alpha$ is such that $90 \%$ of the total energy released is deposited in the first $t_{90}^{*} \mathrm{~s}$ of the simulation, if there were no initial linear increase in the additional heating term up to $t^{*}=t_{\text {rise }}^{*}$, i.e., if $t_{\text {rise }}^{*}=0$. By introducing $t_{\text {dep }}^{*}=t_{90}^{*}+t_{\text {rise }}^{*}$, the amount of energy which is released after $t_{\text {dep }}^{*}$ is $0.9+t_{\text {rise }}^{*} \ln (10) /\left(2 t_{90}^{*}\right)$ times the total amount of energy deposition, $E_{0}$.

\subsection{Numerical setup}

All numerical experiments are performed using a 2D compressible MHD code based on staggered meshes (see Roussev et al. 2001a). A stretching of the numerical grid is applied in order to better resolve the region around the current concentration where the most important dynamics of the experiments is expected to take place. The numerical setup is the same as that presented in Roussev et al. (2001a). In brief, the 2D grid on which the eleven experiments are carried out is of size $N_{x} \times N_{y}=400 \times 700$. The numerical domain is chosen to be of size $\left[2 x_{\max }^{*}, 2 y_{\max }^{*}\right]=[6,8]$. The stretching of the grid is applied in the $x$-direction only (across the current channel), and is such that $50 \%$ of the grid points are located within $-0.7 \leq x^{*} \leq 0.7$. The parameter set used in the present modelling is as follows: $\delta=25, \epsilon=7, \Delta \rho=4.5, \theta=0.6, y_{\mathrm{s}}^{*}=1.6$, $\gamma=1.667, \beta=0.164, M=0.05, t_{\text {rise }}^{*}=0.4$ and $t_{90}^{*}=1.6$.

\section{Discussion on the numerical results}

In the following section we discuss the results obtained from our numerical modelling. The general dynamics shared by all of the experiments is analysed in Sect. 5.1. The observable consequences, in terms of line profiles, are presented in Sect. 5.2. A summary of the results and final conclusions are given in Sect. 6.

\subsection{General dynamics}

In all of the experiments, the energy depositions occur on one and the same dynamic time-scale. The linear increase of the ad hoc heating term is assigned at $t=0$ and operates until $t_{\text {rise }}=0.4 t_{\mathrm{Alfv}}$, corresponding to $6.5 \mathrm{~s}$. The characteristic time of "exponential decay" of the driver term, $t_{90}$, is chosen as $1.6 t_{\mathrm{Alfv}}$, equivalent to $26.5 \mathrm{~s}$.

All energy depositions take place in the current channel along the symmetry axis $x^{*}=0$ of the physical model. Energy release of the same amount (for example experiments A1-4) is initiated at four different locations along $y^{*}$, i.e., $y_{0}^{*}$, characterized by the following values of $\log T$ in the initial background plasma: 4.7, 5.0, 5.3, and 5.6.

With the onset of energy release, both the kinetic gas pressure and temperature start rising, thus creating an excess of pressure at the site of the explosion. As the pressure gradient grows in time, the plasma heated at the site of energy release expands dynamically into the yet non-affected surrounding plasma. Furthermore, the onset of this small-scale transient event also initiates a fast MHD wave that propagates away from the site of the explosion. 

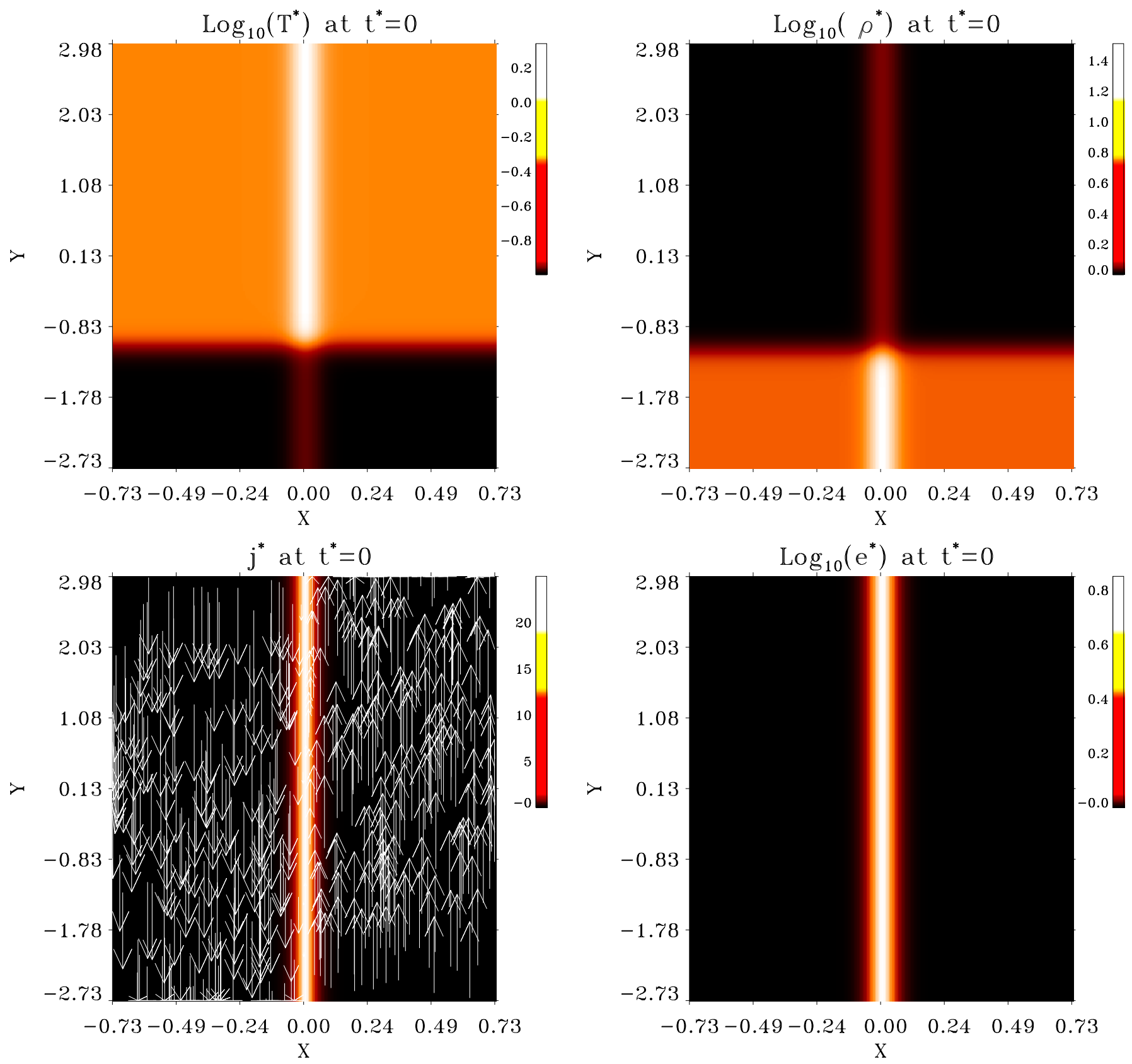

Fig. 4. The initial state for temperature (top left), mass density (top right), current density and magnetic field topology (bottom left) and thermal energy (bottom right).

Since the plasma beta parameter outside the current "sheet" is less than unity (i.e., $\beta=0.164$ ), the increased plasma pressure at the site of the explosion can be balanced only by a small perturbation of the Lorentz force in the direction perpendicular to the current concentration. The plasma expansion therefore occurs mainly along the magnetic field lines, and thus the heated plasma spreads preferably along the current concentration. This does not imply that similar results may be obtained if the localized energy release were to occur in a simple magnetic loop without the current concentration since the plasma beta parameter goes to infinity as one approaches the field reversal line, $x^{*}=0$. This means that exactly in the mid-plane of the current sheet the increased kinetic gas pressure cannot be balanced by the very small Lorentz force, unlike the situation where there is a unipolar region. As one moves outward from the mid-plane of the current sheet, the plasma beta decreases and the magnetic pressure becomes more and more dominant. Right outside the current structure, the plasma beta parameter approaches its value at large distances, i.e. 0.164. This means that if the kinetic gas pressure increases by say $100 \%$, this would be balanced by a 16.4 percent increase in the magnetic pressure.

In addition, the effect of field-aligned thermal conduction is to expand the enhanced temperature region along the magnetic field lines even further than just a pure expansion (nearly adiabatic) of hot plasma. As an increasing amount of energy 
is deposited in the system, the peak temperature at the site of the explosion continues rising as a function of time. This occurs because of a combination of several factors. In the first place, the time-dependent increase in temperature initiates the expansion of heated plasma, which locally leads to a decrease in mass density. As the mass density decreases, the radiative losses $\left(N_{\mathrm{e}}^{2}\right.$ dependence) decrease too, while the temperature increase goes even further before a balance can be reached. Secondly, since the background heating, $S^{*}$, is maintained at the same level, while the mass density decreases in time, the same amount of energy input will support a higher steady-state of the plasma temperature. This is therefore a run-away process which is slowed down by the combined effect of plasma expansion, field-aligned heat conduction, and exponential decrease of the energy input with time.

As the region of enhanced temperature expands, the plasma surrounding the current concentration is pushed away. Since an increasing amount of energy is deposited into the system, the hot plasma blown out from the site of the explosion is dynamically accelerated and bow shocks are subsequently formed at the locations where the unperturbed plasma is being hit. The initial physical conditions are such that the sound speed takes maximum values along the symmetry axis, $x^{*}=0$, while the Alfvén velocity is zero here. Comparing the flow velocity with the local sound speed and Alfvén velocity in the vicinity of the current channel around $x^{*}=0$, it appears that this velocity is both supersonic and super-Alfvénic in all of the experiments. One would therefore expect to observe maximum outflow velocities away from the symmetry axis at the locations where the flow becomes sub-Alfvénic rather than along this axis (although the largest pressure gradient is reached here). This is what causes two sets of outflow velocity peaks to appear on each side of the density depletion region. It is found that the peak velocity first increases in time and then starts to decline after reaching some maximum value of $u_{\max }$. The peak flow velocity is found to depend on the amount of energy released, nearly as $\sim E_{0}^{1 / 2}$. Furthermore, the highest velocities of bulk motions are found to be located at the edges of the enhanced temperature region, clearly indicating the direction of plasma expansion.

As time progresses, there are two density features that are dynamically created and evolve in the opposite $y^{*}$-direction. They propagate along $y^{*}$ due to the fact that the magnetic field outside the current concentration acts nearly like rigid walls (low plasma "beta"), and because of the radial expansion of the temperature front moving along the current concentration.

The higher the amount of energy deposition, the denser the outflow feature becomes, as well as the higher the temperature increase at the site of the explosion. Also, the more energy that is deposited into the system, the higher the value of the maximum flow velocity, $u_{\max }$.

The basic difference of the same amount of energy deposition taking place at different initial temperatures is basically on the dynamic time-scale on which the plasma responds to such energy release. In the first place, because of the higher value of the sound speed at increased plasma temperature, the expansion of heated plasma will occur faster, and thus involve a shorter dynamic time-scale. Secondly, the importance of thermal conduction and radiative losses will also vary, with an effect of a slight predominance of the heat conduction over the radiative losses, as being more non-linear with respect to changes in the physical conditions. The higher the initial temperature at the site of explosion, the faster the thermal conduction, as well as it's importance over the radiative losses.

In the next section, it will be shown that all this influence the observational consequences of such micro-scale transient events in various resonance transition region lines formed at different plasma temperatures.

\subsection{Line synthesis}

As pointed out by Joselyn et al. (1979a,b) and more recently by Sarro et al. (1999) and Roussev et al. (2001b), the ionization balance (EI) is a valid approximation only when bulk motions and gradients in the physical parameters of the emitting plasma involve dynamic time-scales that are long compared to the ionization and recombination times. In circumstances where this does not hold true, time-dependent ion populations need to be calculated in order to describe the emitting properties of the plasma more accurately. In the case of non-equilibrium ionization (non-EI), the full time-dependent ion populations are given via

$$
\begin{aligned}
\frac{\partial N_{i}}{\partial t}+u_{y} \cdot \frac{\partial N_{i}}{\partial y^{*}}= & -N_{\mathrm{e}} N_{i}\left[S_{i, i+1}+\alpha_{\mathrm{r}}^{i, i+1}+\alpha_{\mathrm{d}}^{i, i+1}\right] \\
& +N_{\mathrm{e}}\left[N_{i-1} S^{i-1, i}+N_{i+1} \alpha_{\mathrm{r}}^{i+1, i} N_{i+1} \alpha_{\mathrm{d}}^{i+1, i}\right],
\end{aligned}
$$

where $\alpha_{\mathrm{d}}^{i, i+1}$ is the collisional-dielectronic recombination coefficient from level $i$ to $i+1, \alpha_{\mathrm{r}}^{i, i+1}$ is the radiative recombination coefficient from level $i$ to $i+1, S_{i, i+1}$ is the collisionaldielectronic ionization coefficient from level $i$ to $i+1$, and $N_{i}$ is the number density of ion $i$.

From the numerical perspective, solving Eq. (9) is a rather stiff problem, and there is currently little progress made in computing time-dependent ion populations in 2D. In the 1D case, we use a code as presented by Sarro et al. (1999) to solve Eq. (9). Since the experiments discussed in the previous section were done in 2D, we reduced the MHD results to that which can be viewed along a line-of-sight by a spectrograph. This was done by considering as a line-of-sight the symmetry axis $x^{*}=0$, and averaging the 2D MHD solution over 19 grid points in the $x^{*}$-direction (in symmetrical manner with respect to $x^{*}=0$ ). This way we obtained a 1D MHD solution along the line-of-sight (only $y^{*}$-dependence), that represents a region 1 arcsec wide in the $x^{*}$-direction, as scaled on the Sun. The 1D code uses as input, the values of $N_{\mathrm{e}}, T$, and $u_{y}$ provided by the simulated 1D MHD solutions.

Once the ion populations of a given element are computed using this 1D code, the emissivity of a given emission line per unit interval of wavelength in an optically thin, collisionally excited resonance line can be obtained from

$E_{\lambda} \propto \frac{h c}{\lambda} \frac{\Omega}{\omega} \frac{N_{1}}{N_{\text {ion }}} \frac{N_{\text {ion }}}{N_{\text {elem }}} \frac{N_{\text {elem }}}{N_{\mathrm{H}}} N_{\mathrm{H}} N_{\mathrm{e}} \frac{\exp \left(-\frac{W}{k_{\mathrm{B}} T}\right)}{\sqrt{T}} \phi(\lambda)$. 
Table 1. The current set of energy depositions experiments.

\begin{tabular}{llll}
\hline \hline$E_{0}$, [ergs] & $4 \times 10^{24}$ & $1 \times 10^{25}$ & $4 \times 10^{25}$ \\
\hline $\log T=4.7$ & A1 & B1 & C1 \\
$\log T=5.0$ & A2 & B2 & C2 \\
$\log T=5.3$ & A3 & B3 & C3 \\
$\log T=5.6$ & A4 & B4 & - \\
\hline
\end{tabular}

Here $h$ is the Planck constant, $c$ is the speed of light, $\Omega$ is the Maxwellian averaged collisional strength, $\omega$ is the statistical weight of the lower level, $N_{1} / N_{\text {ion }}$ is the ratio of ions responsible for the emission in the ground state relative to the total number of ions per unit volume, $N_{\text {ion }} / N_{\text {elem }}$ is the relative population of the ion, $N_{\text {elem }} / N_{\mathrm{H}}$ is the element abundance, $N_{\mathrm{H}}$ is the proton density, $N_{\mathrm{e}}$ is the electron density, $W$ is the energy difference between the upper and lower levels, $k_{\mathrm{B}}$ is the Boltzmann constant, $T$ is the temperature, and

$\phi(\lambda)=\frac{\exp -\left[\frac{\left(\lambda-\lambda_{0}-\lambda_{\mathrm{s}}\right)}{\Delta \lambda_{0}}\right]^{2}}{\Delta \lambda_{0} \sqrt{\pi}}$

is the line profile. In the above definition of $\phi(\lambda), \lambda_{0}$ is the rest wavelength of the resonance line and $\lambda_{\mathrm{s}}=v_{\mathrm{p}}\left(\lambda-\lambda_{0}\right) / c$ is the Doppler-shift corresponding to the velocity of the plasma projected on the line-of-sight, $v_{\mathrm{p}}$. The Doppler width of the line, $\Delta \lambda_{0}$, is assumed to be due to thermal motions only, and is given by

$\Delta \lambda_{0}=\frac{\lambda_{0}}{c} \sqrt{\frac{2 k_{\mathrm{B}} T}{m_{i}}}$

where $m_{i}$ is the mass of the ion emitting in the resonance line of interest.

By knowing the distribution of emissivities along the lineof-sight, the total intensity can be computed as

$I(\lambda, t)=\int_{-y_{\max }^{*}}^{y_{\max }^{*}} E\left(\lambda, y^{\prime}, t\right) \mathrm{d} y^{\prime}$,

where $2 y_{\max }^{*}$ is the total length of the emitting source projected on the line-of-sight. We use the above to perform line synthesis of the resonance lines of C IV $1548 \AA$, O VI $1032 \AA$, and Ne vIII $770 \AA$, as discussed below.

In Fig. 5 we show sample line profiles for C IV $1548 \AA$, O vi $1032 \AA$ and Ne vIII $770 \AA$ for the case of an energy deposition of $1 \times 10^{25} \mathrm{ergs}$ at $2 \times 10^{5} \mathrm{~K}$. The simulations end at $192 \mathrm{~s}$ and on this figure we plot 6 line profiles with a timedifference of $35 \mathrm{~s}$ each. Thus, the last line profile will correspond to $t=175 \mathrm{~s}$. The time evolution may be more clearly seen in Fig. 6, where we show the line-shift as a function of time for two different energy depositions, namely $1 \times 10^{25} \mathrm{erg}$ and $4 \times 10^{25} \mathrm{erg}$ at all four locations. Each line is discussed in detail below.
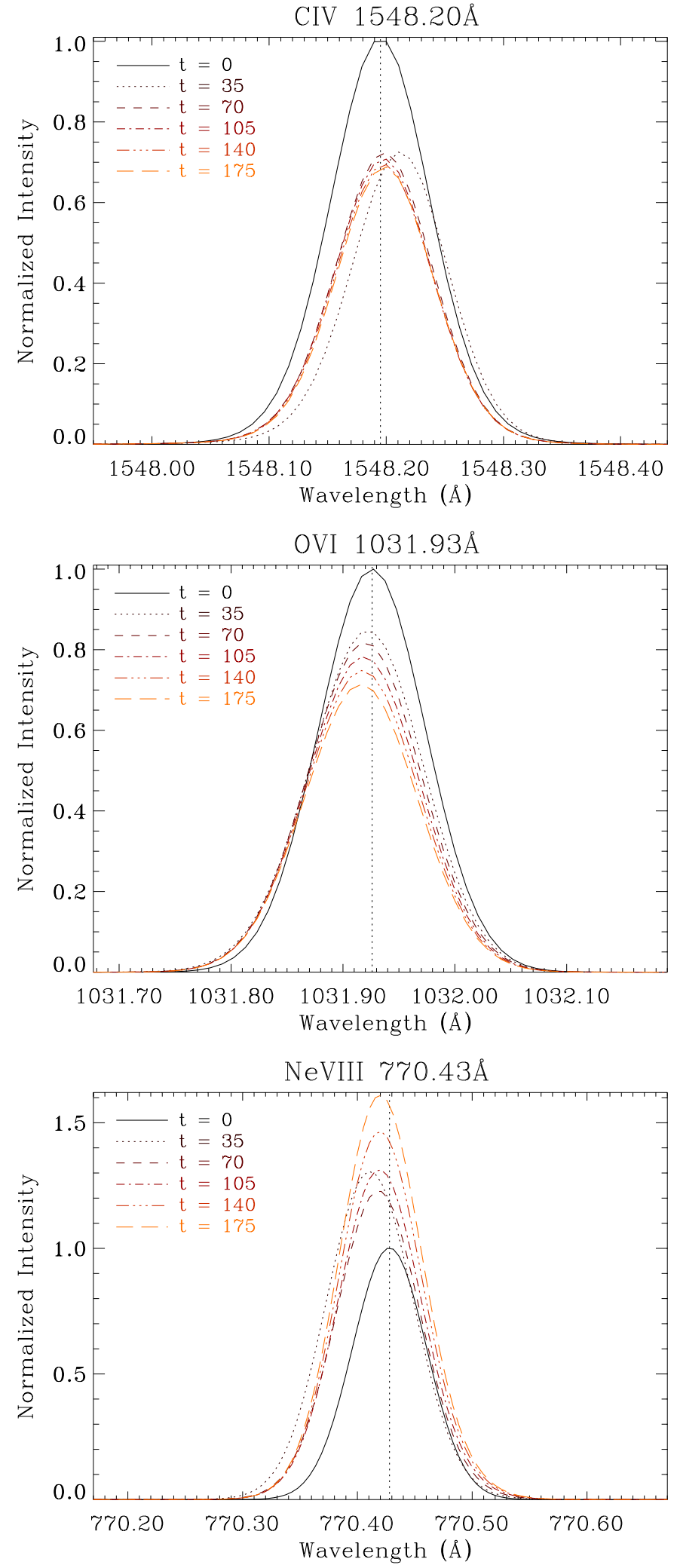

Fig. 5. Sample line profiles for C IV $1548 \AA$ (upper panel), O vi $1032 \AA$ (middle panel) and Ne vIII $770 \AA$, respectively, for the case of an energy deposition of $1 \times 10^{25} \mathrm{ergs}$ at $2 \times 10^{5} \mathrm{~K}$. The simulations for this case end at $t=192 \mathrm{~s}$ and on this figure we plot 6 line profiles with a time-difference of $35 \mathrm{~s}$ each. 

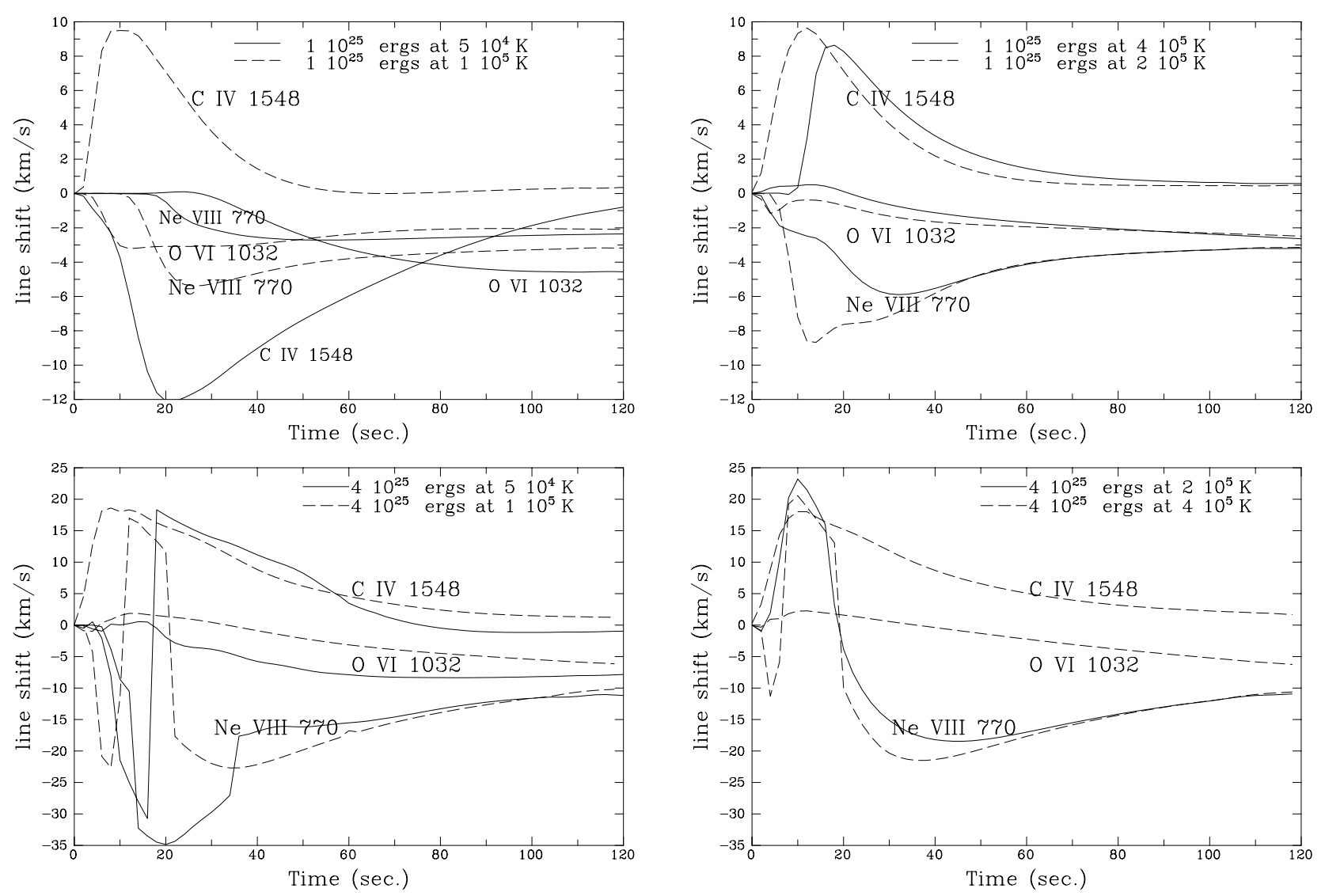

Fig. 6. Line shift, in $\mathrm{km} \mathrm{s}^{-1}$, for an energy deposition of $1 \times 10^{25} \mathrm{ergs}$ at $5 \times 10^{4} \mathrm{~K}$ and $1 \times 10^{5} \mathrm{~K}$ (top left panel), an energy deposition of $1 \times 10^{25}$ ergs at $2 \times 10^{5} \mathrm{~K}$ and $4 \times 10^{5} \mathrm{~K}$ (top right panel), an energy deposition of $4 \times 10^{25}$ ergs at $5 \times 10^{4} \mathrm{~K}$ and $1 \times 10^{5} \mathrm{~K}$ (bottom left panel), and an energy deposition of $4 \times 10^{25}$ ergs at $2 \times 10^{5} \mathrm{~K}$ and $4 \times 10^{5} \mathrm{~K}$ (bottom right panel) for C IV $1548 \AA$, O vi $1032 \AA$ and Ne vIII $770 \AA$.

\subsubsection{The C Iv line}

In all experiments with index "2", i.e., A2-B2-C2 (see Table 1), the energy depositions take place at a temperature of the initial background plasma that is rather close to the temperature of formation of the C IV $1548 \AA$ resonant line. In all other experiments, the energy release happens at higher temperature, i.e., $\log T=5.3$ and 5.6, or in the case of index " 1 " experiments at a lower temperature.

The behavior of the $\mathrm{C}$ IV line in all experiments where the energy deposition take place at the same initial temperature is rather similar. The main difference is in the maximum value of red-shift reached in each individual experiment.

In the A1 and B1 experiments, we initially see blue-shifted plasma up until $\sim 120 \mathrm{~s}$, there-ward it is zero. The " $\mathrm{C} 1$ " experiment initially shows two jets, blue and red with the blue being dominant for the first $20 \mathrm{~s}$. Thereafter, the red-shift is dominant, decreasing from a maximum value of $\sim 17 \mathrm{~km} \mathrm{~s}^{-1}$ to zero after $80 \mathrm{~s}$. Thus for higher energy deposition, the line will always be mostly red-shifted. In A2, B2, and C2 experiments, the time series show an increasing Doppler shift of the line profile towards the red part of the spectra up to a certain time (approx. $10 \mathrm{~s}$ ), followed by a turn-over point, and then a gradual shift back to some small amount of red-shift at the end of the time series. The latter ends at times: 202 s (A2), 192 s (B2), and $118 \mathrm{~s}(\mathrm{C} 2)$, respectively. By comparing these cases, the main conclusion is: the higher the amount of energy deposition, the higher the peak value of red-shift. The latter is derived to be $3.7 \mathrm{~km} \mathrm{~s}^{-1}$ (A2), $9.5 \mathrm{~km} \mathrm{~s}^{-1}$ (B2), and $18.6 \mathrm{~km} \mathrm{~s}^{-1}$ (C2), respectively. At the end of the time series for these three cases, there is a non-zero red-shift of the order of $0.4 \mathrm{~km} \mathrm{~s}^{-1}$ (A2), $0.7 \mathrm{~km} \mathrm{~s}^{-1}$ (B2), and $1.2 \mathrm{~km} \mathrm{~s}^{-1}(\mathrm{C} 2)$, respectively.

A similar behavior in the time series is also seen in the experiments where the energy deposition takes place at $\log T=$ 5.3 , i.e., experiments $\mathrm{A} 3, \mathrm{~B} 3$, and $\mathrm{C} 3$. The peak values of red-shift are reached at a slightly later time than in the previous case, i.e., approx. $12 \mathrm{~s}$. The main difference is then found to occur in the value of this maximum red-shift, i.e., $5.2 \mathrm{~km} \mathrm{~s}^{-1}$ (A3), $9.6 \mathrm{~km} \mathrm{~s}^{-1}$ (B3), and $18.0 \mathrm{~km} \mathrm{~s}^{-1}$ (C3), respectively. The time series relevant to these three particular experiments end at the same time as the corresponding ones of the previous three, i.e., $202 \mathrm{~s}$ (A3), $192 \mathrm{~s}$ (B3), and $118 \mathrm{~s}$ (C3), respectively. The overall red-shift reached at the end of the time series, is of the order of $0.4 \mathrm{~km} \mathrm{~s}^{-1}$ (A3), $0.8 \mathrm{~km} \mathrm{~s}^{-1}$ (B3), and $1.7 \mathrm{~km} \mathrm{~s}^{-1}(\mathrm{C} 3)$, respectively.

In $\mathrm{A} 4$ and $\mathrm{B} 4$, the energy is released at $\log T=5.6$. Comparing with the previous two sets of experiments, here we again find a similar trend in the response of the $C$ Iv line to such small-scale energy deposition. The maximum value of 
red-shift is reached at an even later time than in the previous two cases, and corresponds to approx. $18 \mathrm{~s}$. The values of redshift at this instant of time are, respectively, $4.5 \mathrm{~km} \mathrm{~s}^{-1}$ (A4), and $8.6 \mathrm{~km} \mathrm{~s}^{-1}$ (B4). At the end of the time series there is again a net red-shift, which is of the same value as in the previous two cases, i.e., $0.4 \mathrm{~km} \mathrm{~s}^{-1}$ (A4), and $0.7 \mathrm{~km} \mathrm{~s}^{-1}$ (B4), respectively.

\subsubsection{The $O$ vı line}

Unlike the $\mathrm{C}$ IV line, the response of the $\mathrm{O}$ VI $1032 \AA$ line is rather interesting. In the $\mathrm{A} 1, \mathrm{~B} 1, \mathrm{C} 1, \mathrm{~A} 2$ and $\mathrm{B} 2$ experiments, the Doppler-shift is towards the blue part of the spectra. On the contrary, the behavior of the former line is mostly in the opposite direction - towards the red part of the spectra. In the A1 and B1 experiments, the maximum value of blue-shift is reached at $t=40 \mathrm{~s}$, and the corresponding values of blue-shift are, respectively, $-1.2 \mathrm{~km} \mathrm{~s}^{-1}$ (A1), and $-2.6 \mathrm{~km} \mathrm{~s}^{-1}$ (B1). In " $\mathrm{C} 1$ " it reaches a maximum blue-shift of $-8.3 \mathrm{~km} \mathrm{~s}^{-1}$ after $80 \mathrm{~s}$.

In the $\mathrm{A} 2$ and $\mathrm{B} 2$ experiments, the maximum value of blue-shift is reached at $t=22 \mathrm{~s}$ (A2), and $t=12 \mathrm{~s}$ (B2), and the corresponding values of blue-shift are, respectively, $-1.7 \mathrm{~km} \mathrm{~s}^{-1}$ (A2), and $-3.2 \mathrm{~km} \mathrm{~s}^{-1}$ (B2). In the $\mathrm{C} 2$ experiment, we first observe a blue-shift in the time series of the $\mathrm{O}$ vI line, alternated with a red-shift at approx $t=6 \mathrm{~s}$. The maximum value of the red-shift is reached at $t=12 \mathrm{~s}$, and corresponds to $1.9 \mathrm{~km} \mathrm{~s}^{-1}$. The red-shift is found to change back to blue-shift at about $t=34 \mathrm{~s}$. In all three experiments A2$\mathrm{B} 2-\mathrm{C} 2$, a net blue-shift of the $\mathrm{O}$ vi line profile is found to be present at the end of the time series. The corresponding values of this blue-shift are $-1.2 \mathrm{~km} \mathrm{~s}^{-1}$ (A2), $-2.2 \mathrm{~km} \mathrm{~s}^{-1}$ (B2), and $-6.1 \mathrm{~km} \mathrm{~s}^{-1}(\mathrm{C} 2)$, respectively.

In experiments $\mathrm{A} 3, \mathrm{~B} 3$, and $\mathrm{C} 3$, where the energy depositions take place at $\log T=5.3$, the $\mathrm{O}$ vi line responds in a rather similar manner to the previous case of $\log T=5.0$. Note that this time the micro-scale explosions occur at a temperature which is rather close to the $\mathrm{O}$ vi line formation temperature. The value of blue-shift in experiments A3 and B3 first increases, reaches some maximum value, decreases, and then starts increasing again. The first peak value in the blue-shift is reached at $t=10 \mathrm{~s}$ for experiment $\mathrm{A} 3$, and $t=4 \mathrm{~s}$ for experiment B3, respectively. The corresponding values of blue-shift are $-1.5 \mathrm{~km} \mathrm{~s}^{-1}$ (A3), $-1.2 \mathrm{~km} \mathrm{~s}^{-1}$ (B3), respectively. In experiment $\mathrm{A} 3$ this is the maximum value of blue-shift reached throughout the time series, while in experiment B3, in turn, the maximum value of blue-shift is observed at the end of the time series. The net blue-shift reached at the end of experiments A3 and B3 is $-1.3 \mathrm{~km} \mathrm{~s}^{-1}$ and $-2.9 \mathrm{~km} \mathrm{~s}^{-1}$, respectively. Similar to the response of $\mathrm{O}$ VI in experiment $\mathrm{C} 2$, in experiment $\mathrm{C} 3$ we again see the appearance of a Doppler-shifted component towards the blue part of the spectra, though occurring at a smaller relative intensity than in the former case, as well as lasting longer (approx. $100 \mathrm{~s}$ ). A red-shift is also seen in the time series that reaches a maximum value of $2.3 \mathrm{~km} \mathrm{~s}^{-1}$ at $t=12 \mathrm{~s}$, subsequently changes into blue-shift at later times. The maximum value of blue-shift is reached at the end of the time series, and corresponds to $-6.3 \mathrm{~km} \mathrm{~s}^{-1}$.
In the case of an energy release taking place at $\log T=$ 5.6, i.e., experiments A4 and B4, first we see red-shifts, which eventually change into blue-shifts at later times. The maximum red-shift observed in experiment A4 is $0.3 \mathrm{~km} \mathrm{~s}^{-1}$, reached at $t=8 \mathrm{~s}$. Similarly, in experiment B4 the corresponding value of red-shift is $0.5 \mathrm{~km} \mathrm{~s}^{-1}$, and is reached at $t=12 \mathrm{~s}$. At $t=16 \mathrm{~s}$ for $\mathrm{A} 4$, and $t=22 \mathrm{~s}$ for $\mathrm{B} 4$, respectively, the red-shift changes into a blue-shift. The values of the latter at the end of the time series are $-1.4 \mathrm{~km} \mathrm{~s}^{-1}$ (A4) and $-3.7 \mathrm{~km} \mathrm{~s}^{-1}$ (B4), respectively.

\subsubsection{The Ne VIII line}

Similar to the $\mathrm{O}$ vi line, the response of the Ne vIII $770 \AA$ line to small-scale energy depositions is also quite interesting. In both experiments A1 and B1, the Doppler-shifts of the $\mathrm{Ne}$ VIII resonant line are all towards the blue part of the spectra. The maximum blue-shift is reached towards the end of the experiment, $\sim 120 \mathrm{~s}$ corresponding to $\sim-1.8 \mathrm{~km} \mathrm{~s}^{-1}$ (A1) and $-4.5 \mathrm{~km} \mathrm{~s}^{-1}$ (B1). In both experiments $\mathrm{A} 2$ and $\mathrm{B} 2$, the Doppler-shift of the Ne viII resonant line are also towards the blue. The maximum value of blue-shift in experiment A2 is reached at the end of the time series, while in experiment B2 this happens at $t=26 \mathrm{~s}$, and the corresponding value of blueshift is $-5.4 \mathrm{~km} \mathrm{~s}^{-1}$. At the end of the time series the blue-shift is $-1.8 \mathrm{~km} \mathrm{~s}^{-1}$ for $\mathrm{A} 2$, and $-3.0 \mathrm{~km} \mathrm{~s}^{-1}$ for $\mathrm{B} 2$, respectively. In experiments $\mathrm{C} 1$ and $\mathrm{C} 2$, the response of the $\mathrm{Ne}$ VIII line is even more complex than that of O VI. In this case, the appearance of two Doppler-shifted components is seen - one in the blue, and another one in the red part of the spectra which appears first, and lasts longer than the blue-shifted component. In $\mathrm{C} 1$, the blue component reaches a maximum value of relative intensity at $t=12 \mathrm{~s}$, and then starts gradually disappearing. The red-shifted component is present until $t=60 \mathrm{~s}$, while thereafter only the blue-shifted plasma remains. The maximum values of blue- and red-shift at which these two components appear are $-40 \mathrm{~km} \mathrm{~s}^{-1}$, and $25 \mathrm{~km} \mathrm{~s}^{-1}$, respectively. The net blue-shift reached at the end of the time series corresponds to $-1.9 \mathrm{~km} \mathrm{~s}^{-1}$.

In $\mathrm{C} 2$, the blue component reaches a maximum value of relative intensity after $t=12 \mathrm{~s}$, and then starts gradually disappearing. The red-shifted component becomes dominant in the spectra approx. $6 \mathrm{~s}$ later than the blue counterpart. The maximum values of blue- and red-shift at which these two components appear are $-40 \mathrm{~km} \mathrm{~s}^{-1}$, and $25 \mathrm{~km} \mathrm{~s}^{-1}$, respectively. The two Doppler components gradually diminish, and after approx. $t=60 \mathrm{~s}$ there is only blue-shifted emission. The net blue-shift reached at the end of the time series is $-4.5 \mathrm{~km} \mathrm{~s}^{-1}$.

In the case where the energy deposition takes place at $\log T=5.3$, i.e., experiments A3 and B3, the dynamic response of the $\mathrm{Ne}$ vIII line is rather similar to that of the previous case. Note that in both cases the energy deposition takes place at a temperature that is below the temperature of formation of the Ne vIII line. The maximum value of blue-shift in experiment A3 is reached at the end of the time series, and corresponds to $-1.8 \mathrm{~km} \mathrm{~s}^{-1}$. In experiment $\mathrm{B} 3$, in turn, the peak value of blue-shift is obtained at $t=14 \mathrm{~s}$, and corresponds to $-8.7 \mathrm{~km} \mathrm{~s}^{-1}$. The blue-shifted plasma at the end of the time 
series is $-2.8 \mathrm{~km} \mathrm{~s}^{-1}$. The two Doppler-shifted components found in the spectra of the $\mathrm{Ne}$ VIII line relevant to experiment $\mathrm{C} 2$ are again present in the spectra for the case of experiment $\mathrm{C} 3$. Just like before, the blue-shifted component appears first and lasts longer, although the dominant relative intensity comes from the red-counterpart. The two components also appear at slightly higher absolute values of Doppler-shift than in the previous case and these are, respectively, $-50 \mathrm{~km} \mathrm{~s}^{-1}$, and $30 \mathrm{~km} \mathrm{~s}^{-1}$. These components disappear from the spectra at almost the same time as in the previous case, i.e., at approx. $t=60 \mathrm{~s}$. There is again a blue-shifted line profile at the end of the time series. The value of blue-shift is of the order of $-10.6 \mathrm{~km} \mathrm{~s}^{-1}$.

In the last two experiments A4 and B4, the peak value of blue-shift is reached at $t=24 \mathrm{~s}$ for experiment A4, and $t=32 \mathrm{~s}$ for experiment B4, respectively. The corresponding values of blue-shift are $-1.7 \mathrm{~km} \mathrm{~s}^{-1}$ (A4), and $-5.9 \mathrm{~km} \mathrm{~s}^{-1}$ (B4), respectively. At the end of the time series, there is an overall blue-shift in both cases that corresponds to a value of $-1.8 \mathrm{~km} \mathrm{~s}^{-1}$ for experiment $\mathrm{A} 4$, and $-3.1 \mathrm{~km} \mathrm{~s}^{-1}$ for experiment $\mathrm{B} 4$.

\section{Conclusions and future work}

In the present simulations, we use small-scale energy depositions to study the effect on observable parameters such as line profiles, line-shifts and intensities. The main conclusion following the $\mathrm{C}$ IV simulations is that the higher the temperature at which the energy deposition takes place, the later the response of the line. In particular, the peak value of red-shift is reached at a later time if the energy release occurs at a higher temperature of the initial background plasma. For $\mathrm{C}$ iv, the maximum red-shift varies between 4 and $19 \mathrm{~km} \mathrm{~s}^{-1}$ for a variety of energy depositions and temperatures in the atmosphere. Towards the end of each simulation, the line-shift approaches zero.

From the $\mathrm{O}$ vi results, one can conclude that, as the energy deposition takes place at increasing initial plasma temperatures, there is a turn-over point at which the blue-shift of the line profile changes into red-shift at early times. Furthermore, the more energy is released, the more complex the related changes in the spectra. In particular, at some high value of energy deposition, more than one Doppler-shifted component start to be present in the spectra. For O VI $1032 \AA$ and for energy deposition below the line formation temperature, we have a blue-shift ranging from $-2 \mathrm{~km} \mathrm{~s}^{-1}$ to $-8 \mathrm{~km} \mathrm{~s}^{-1}$.

For Ne vIII, only blue-shifts are observed in the time series. This is because in all these cases the initial temperature at the site of the explosion is either below or close (experiments A4 and B4) to the temperature of formation of the Ne VIII line. Just like in the case of the $\mathrm{O}$ vI line, at some high value of the energy deposition, Doppler-shifted components start to be present in the spectra. This time, however, two components are seen instead of only one in the case of $\mathrm{O}$ VI.

The intensity variations resulting from these experiments are quite different from an earlier study by Teriaca et al. (1999b) and Teriaca \& Doyle (2001). Here, we have a very different initial state, e.g., the above paper had a coronal loop but here there is a magnetic field reversal region. Secondly, the distribution of physical variables along the loop in the above work is different than that in this model. Thirdly, the previous modelling was a $1 \mathrm{D}$ hydrodynamic simulation, while these are 2D MHD.

Comparing the $\mathrm{C}_{\text {IV }}$ modelling results with the $\mathrm{C}_{\text {III }} 977 \AA$ observations (the $\mathrm{C}$ III line is formed at $80000 \mathrm{~K}$ compared with $\mathrm{C}$ iv at $100000 \mathrm{~K}$ ), we see that there is good overall agreement. The observed time-averaged C III line-shift varies between 2 and $20 \mathrm{~km} \mathrm{~s}^{-1}$ depending on the amount of magnetic activity, similar to the $\mathrm{C}$ Iv line simulations. The highly variable nature of the observed line-shift could be re-produced via a series of energy releases (of the order of magnitude as given in Table 1), occurring approximately every 20 to $60 \mathrm{~s}$. with the most of these being low energy events, probably less than $4 \times 10^{24} \mathrm{erg}$. On the other hand, the agreement between the $\mathrm{O}$ vi simulations and observations is poor, with the simulations indicating an average blue-shifted plasma of a few $\mathrm{km} \mathrm{s}^{-1}$ while the observations show a slightly red-shifted plasma. For Ne vIII, both the internetwork and network observational data imply an up-flow, in agreement with the models. However, the bright network observations show a down-flow. This could indicate that the bright network is the region where spicular down-flows are dominant and therefore the simple model presented here is more applicable to the internetwork and "normal" network regions.

Acknowledgements. Research at Armagh Observatory is grant-aided by the N. Ireland Dept. of Culture, Arts and Leisure. We are very thankful to K. Galsgaard for useful comments and suggestions made while the manuscript was prepared, to R. Erdélyi for initial discussions and K. Curdt for discussions on the wavelength calibration. This work was in part supported by PPARC grants PPA/G/S/1999/00055, PPA/V/S/1999/00628 and PPA/V/S/1999/00668 while the SUMER project is financially supported by DLR, CNES, NASA, and PRODEX.

\section{References}

Banerjee, D., O’Shea, E., Doyle, J. G., \& Goossens, M. 2001, A\&A, 371,1137

Brekke, P., Hassler, D. M., \& Wilhelm, K. 1997, Sol. Phy., 175, 349

Chae, J., Yun, H. S., \& Poland, A. I. 1997, ApJ, 480, 817

Chae, J., Yun, H. S., \& Poland, A. I. 1998, ApJS, 114, 151

Dammasch, I. E., Wilhelm, K., Curdt, W., \& Hassler, D. M. 1999, A\&A, 346, 285

Doyle, J. G., van den Oord, G. H. J., O'Shea, E., \& Banerjee, D. 1998, Sol. Phys., 181, 51

Hansteen, V. H. 1993, ApJ, 402, 741

Hansteen, V. H., \& Wikst $\varnothing 1$, O. 1994, A\&A, 290, 995

Hansteen, V. H., Maltby, P., \& Malagoli, A. 1977, ASP Conf. Ser., 111, ed. R. D. Bentley, \& J. T. Mariska, 116

Hansteen, V. H., Betta, R., \& Carlsson, M. 2000, A\&A, 360, 742

Lemaire, P., Wilhelm, K., \& Curdt, W., et al. 1997, Sol. Phys., 170, 105

Joselyn, J. A., Munro, R. H., \& Holzer, T. E. 1979a, Sol. Phys., 64, 57 Joselyn, J. A., Munro, R. H., \& Holzer, T. E. 1979b, ApJS, 40, 793

Mariska, J. T., \& Boris, J. P. 1983, ApJ, 267, 409

Mariska, J. T. 1988, ApJ, 334, 489

Mariska, J. T. 1992, The Solar Transition Region (Cambridge University Press, Cambridge)

Peter, H. 2000, A\&A, 360, 761 
Peter, H. 2001, A\&A, 374, 1108

Peter, H., \& Judge, P .G. 1999, ApJ, 522, 1148

Pneuman, G. W., \& Kopp, R. A. 1978, Sol. Phys., 57, 49

Reale, F., Peres, G., \& Serio, S. 1997, A\&A, 318, 506

Roussev, I., Galsgaard, K., Erdélyi, R., \& Doyle, J. G. 2001a, A\&A, 370,298

Roussev, I., Doyle, J. G., \& Galsgaard, K., \& Erdélyi, R. 2001b, A\&A, 380, 719

Sarro, L. M., Erdélyi, R., Doyle, J. G., \& Perez, E. P. 1999, A\&A, 351, 721

Spadaro, D., Antiochos, S. K., \& Mariska, J. T. 1991, Sol. Phys., 382, 338
Teriaca, L., Banerjee, D., \& Doyle, J. G. 1999a, A\&A, 349, 636

Teriaca, L., Doyle, J. G., Erdélyi, R., \& Sarro, L. M. 1999b, A\&A, 352, L99

Teriaca, L., Doyle, J. G., Erdélyi, R., Sarro, L. M., \& Banerjee, D. 1999c, Proc. 9th European Meet. on Sol. Phys., ESA SP-448, 379

Teriaca, L., \& Doyle, J. G. 2001, Ap\&S, 259, 307

Wikstøl, Ø., Hansteen, V. H., Carlsson, M., \& Judge, P. G. 2000, ApJ, 531,1150

Wilhelm, K., Curdt, W., Marsch, E., et al. 1995, Sol. Phys., 162, 189 Wilhelm, K. 2000, A\&A, 360, 351 